\title{
IS THE SLIT OF A RATIONAL SLIT MAPPING IN $S$ STRAIGHT?
}

\author{
URI SREBRO
}

\begin{abstract}
The question in the title is answered by showing that if $f$ is a rational function in $\hat{\mathbf{C}}$ and maps some disk injectively onto the complement of a set $E$ of empty interior, then degree $(f)=2$, and $E$ is either a circular arc or a line segment in $\hat{\mathbf{C}}=\mathbf{C} \cup\{\infty\}$.
\end{abstract}

Here $S$ denotes the class of all univalent analytic functions $f(z)=z+a_{2} z^{2}+\cdots$ in the unit disc $\Delta=\{z \in \mathbf{C}:|z|<1\}$. A function $f$ in $S$ is called a slit mapping if $\Gamma=\hat{\mathbf{C}}-f(\Delta)$ is a Jordan arc in $\hat{\mathbf{C}}=\mathbf{C} \cup\{\infty\}$.

The question in the title arose in the work of Duren, Leung and Schiffer [2]. It had been conjectured earlier by $\mathrm{D}$. Wilken that the answer to the question is positive. A positive answer for the case $f(z)=z / P(z)$, where $P$ is a polynomial, follows from [1].

The following theorem shows that the answer is always positive, as conjectured, and that every rational slit mapping in $S$ is necessarily of the form

$$
f(z)=A\left[\left(\frac{z-a}{z-b}\right)^{2}-\left(\frac{a}{b}\right)^{2}\right]
$$

for some distinct points $a, b$ in $\partial \Delta$ and some constant $A$ for which $f^{\prime}(0)=1$, or equivalently, $f=\varphi \circ k \circ \psi$, where $k(z)=z /(1-z)^{2}$ is the Koebe function and $\varphi$ and $\psi$ are Möbius transformations.

THEOREM. Let $f$ be a rational function in $\mathbf{C}$ of degree $n$. If $f_{0}=f \mid \Delta$ is injective and $E=\hat{\mathbf{C}}-f(\Delta)$ has empty interior, then

(i) $n=2$,

(ii) $E$ is either a circular arc or a line segment in $\hat{\mathbf{C}}$,

(iii) $f(z)=\varphi\left(((z-a) /(z-b))^{2}\right)$ for some distinct points $a, b \in \partial \Delta$ and some Möbius transformation $\varphi$ with $\varphi(0)=f(a)$ and $\varphi(\infty)=f(b)$.

Proof. Since $f$ is continuous on $\bar{\Delta}$ and a homeomorphism on $\Delta$ and since int $E=\varnothing$, it follows that $f(\partial \Delta)=\partial f(\Delta)=E$. Now, $f$ is meromorphic on $\partial \Delta$; hence it has finitely many branch points on $\partial \Delta$, and therefore $E=f(\partial \Delta)$ is a finite union of analytic arcs.

Let $\psi(z)=1 / \bar{z}$. Then $g=f \circ \psi \circ f_{0}^{-1}$ is antimeromorphic in $D=f(\Delta)$ and has a

Received by the editors March 1, 1982.

1980 Mathematics Subject Classification. Primary 30C55, 30C25, 30C35.

Key words and phrases. Univalent functions, slit functions, Koebe functions, rational functions. 
continuous extension on $\bar{D}=\hat{\mathbf{C}}$, denoted again by $g$, with $g(w)=w$ for all $w \in \partial D$ $=E$. Since $E$ is a finite union of analytic arcs, and since $\frac{g(z)}{g(s}$ continuous on $\hat{\mathbf{C}}$ and meromorphic in $\hat{\mathbf{C}}-E$, it follows (cf. [3, p. 183]) that $\overline{g(z)}$ is meromorphic in $\hat{\mathbf{C}}$ and $g$ is antimeromorphic in $\hat{\mathbf{C}}$. Therefore, $h=g \circ g$ is meromorphic in $\hat{\mathbf{C}}$, and $h(w)=w$ for all $w \in E$. Hence, $h(w)=w$ for all $w \in \hat{\mathbf{C}}$, and thus $g$ is an involution. Therefore $g$ and, consequently, $f_{1}=f \mid \hat{\mathbf{C}}-\bar{\Delta}$ are injective. But $f_{0}$ is injective too. Hence, $n \leqslant 2$, and since $f$ is not a Möbius transformation, (i) follows.

It is well known that a rational function $f$ of degree two has exactly two distinct branch points $a$ and $b$, each of order one, with $f(a) \neq f(b)$. This follows from the Riemann-Hurwitz formula or can be verified by counting the number of multiple poles of $f(z)=\left(a z^{2}+b z+c\right) /\left(A z^{2}+B z+C\right)$ and the number of zeros of $f^{\prime}(z)$ and by noting that $f(a)=f(b)$ is ruled out since degree $(f)=2$. Now for $\varphi$ as in (iii), $F=\varphi^{-1} \circ f$ is rational of degree two with a double zero at $a$ and a double pole at $b$. Hence, $F(z)=A((z-a) /(z-b))^{2}$ for some $A \neq 0$. Clearly, $a, b \in \partial \Delta$ since $f_{0}$ and $f_{1}$ are injective. Thus (iii) and then (ii) follow.

REMARK. When this paper was in print the author found out that an affirmative answer to the question in the title was given already by L. Fuchs in 1874 (J. Reine Angew. Math. 77, 339-352), that another proof was given by R. Stolzenberg in 1928 (Jber. Deutsch. Math.-Verein. 37, 64-68) and that it was rediscovered (with still another proof) by L. Bieberbach in 1940 (Viert. Natursfor. Ges. Zürich 85, 143-148). The proof here is different from the previous ones.

Fuchs asserted in his paper that the result holds for algebraic functions. There is, however, an error in his arguments and the question in the title with the word "rational" replaced by the word "algebraic" remains open.

ACKNOWLEDGEMENT. I wish to thank Burt Rodin for telling me about the question and Peter Duren, Carl FitzGerald and Steve Warschawski for helpful comments.

\section{REFERENCES}

1. D. Aharonov, A note on slit mappings, Bull. Amer. Math. Soc. 75 (1969), 836-839.

2. P. L. Duren, Y. J. Leung and M. M. Schiffer, Support points with maximum radial angle, Complex Variables, Theory Appl. 1 (1982/83), 263-277.

3. Z. Nehari, Conformal mapping, McGraw-Hill, New York, 1952.

Department of Mathematics, University of California, San Diego, la Jolla, California 92093

Current address: Department of Mathematics, Technion, Haifa 32000, Israel 